\title{
Simultaneous Multiple Rotation Averaging using Lagrangian Duality
}

\author{
Johan Fredriksson and Carl Olsson \\ Centre for Mathematical Sciences \\ Lund University, Sweden
}

\begin{abstract}
Multiple rotation averaging is an important problem in computer vision. The problem is challenging because of the nonlinear constraints required to represent the set of rotations. To our knowledge no one has proposed any globally optimal solution for the case of simultaneous updates of the rotations. In this paper we propose a simple procedure based on Lagrangian duality that can be used to verify global optimality of a local solution, by solving a linear system of equations. We show experimentally on real and synthetic data that unless the noise levels are extremely high this procedure always generates the globally optimal solution.
\end{abstract}

\section{Introduction}

Rotation averaging is an important sub-problem in computer vision applications such as structure from motion and panoramic stitching. While it would be desirable to solve the structure from motion problem in one single step no such solution exists. In practice we are limited to local optimization from some suitably selected initial solution. The most commonly used approach for initialization is perhaps sequential reconstruction using minimal solvers such as [1]. Other alternatives are e.g. factorization approaches [2], hierarchical reconstruction [3], discrete [4] and continuous global optimization approaches [5]. Multiple rotation averaging is a sub-problem in the reconstruction pipeline. It aims at computing orientations for all the cameras, with respect to some common coordinate system, from relative rotation estimates between pairs of cameras that have been obtained using for example [1]. The problem is made difficult by the non-linear constraints required to represent the set of rotations. It has been studied in several recent works e.g. [6-9, 5, 10-12].

In [6] Govindu showed that when representing the rotations with quaternions the problem can be viewed as a linear least squares problem. However, since a quaternion only represents a rotation if its length is one, an additional norm constraint is obtained for each rotation. In [6] these are disregarded and the problem is solved as a regular least squares problem. Martinec and Pajdla [9] noted that in some cases the norms obtained from the least squares problem were far from one, which resulted in poor estimations. They instead used the matrix representation of rotations and solved a linear system without enforcing rotational constraints. 
For handling outliers among the relative rotations graph methods can be utilized. These methods use a so called camera graph, where the nodes are the cameras and the edges are the relative rotations. In [8] a sampling scheme is proposed where a minimal number of relative rotations needed for computing the absolute orientations are randomly sampled in each iteration. This can be seen as sampling spanning trees in the camera graph. Enqvist etal [5] start from a maximal spanning tree and add relative rotations that are consistent with this solution. Zach etal [13] use a rather involved Bayesian model to detect inconsistent cycles in the camera graph. In [14] cycles are also used to detect inconsistent cycles.

In [10] the Wieiszfeld algorithm is applied to single rotation averaging with the $L_{1}$ norm. In [11] convexity properties of the single rotation averaging problem are given. Unfortunately these results do not seem to extend to multiple rotation averaging. To our knowledge no globally optimal solution has been proposed for this case.

In this paper we propose a framework for optimizing the least squares problem from [6] while simultaneously incorporating the norm constraints of the quaternions. We show experimentally that for the majority of cases it is possible to find a solution that is guaranteed to be globally optimal. This approach is similar in spirit to [15] where global optimality is verified for a candidate local minimum, however the method is very different.

In summary our main contributions are:

- Based on duality we derive a semi definite program that gives a lower bound on our original problem. We show that unless the noise level is exceptionally large the lower bound is tight and can be used to find guaranteed optimal solutions.

- To avoid solving the costly semi definite program we show how to compute the lower bound from a candidate solution, which gives a significant speedup.

- We verify that if the noise level is moderate the approach from [6] provides a good solution.

\section{Rotation Averaging}

Rotation averaging as approached by [6] can be seen as the process of determining absolute orientations from relative rotation estimates. Using for example epipolar geometry we can determine approximate relative rotations between all camera pairs. Rotation averaging attempts to compute the absolute orientations for the cameras with respect to some common coordinate system. Under ideal (noiseless) conditions the absolute and relative rotations are related through the equations

$$
R_{i j} R_{i}=R_{j}
$$

where $R_{i j}$ is the relative rotation estimate and $R_{i}$ and $R_{j}$ are the absolute orientations. Under the assumption of approximately Gaussian noise, the goal is to solve (1) in a least squares scene. This problem is difficult because of the 
nonlinear constraints arising from the requirement that the $R_{i}$ are rotations. In [6] the problem is formulated using quaternions as

$$
\min \sum_{i j}\left\|Q_{i j} \mathbf{q}_{i}-\mathbf{q}_{j}\right\|^{2}
$$

where $Q_{i j}$ is a $4 \times 4$ orthogonal matrix representing the relative rotation and the $4 \times 1$ vectors $\mathbf{q}_{i}, \mathbf{q}_{j}$ are quaternion representations of the absolute orientations. Since the norm of the quaternions should be one, (2) can be equivalently written

$$
\max \sum_{i j} \mathbf{q}_{j}^{T} Q_{i j} \mathbf{q}_{i}
$$

By adding the constraint $\sum_{i}\left\|\mathbf{q}_{i}\right\|^{2}=n$, where $n$ is the number of quaternions, this turns into a homogeneous quadratic minimization problem. Therefore [6] proposes to solve this problem using eigenvalue computations. We will refer to this problem as the eigen-solution.

This formulation has two problems. First, a rotation can always be represented using two quaternions $\mathbf{q}_{i}$ and $-\mathbf{q}_{i}$. Therefore, if the sign of one of the quaternions is chosen incorrectly the term $\left\|Q_{i j} \mathbf{q}_{i}-\mathbf{q}_{j}\right\|$ might be large even though the rotations are similar. To be correct the error should really be measured by

$$
\sum_{i j} \min \left(\left\|Q_{i j} \mathbf{q}_{i}-\mathbf{q}_{j}\right\|^{2},\left\|Q_{i j} \mathbf{q}_{i}+\mathbf{q}_{j}\right\|^{2}\right)
$$

see [10]. On the other hand the quaternion formulation reduces the number of nonlinear constraints that need to be enforced. Furthermore, the correct sign of the quaternion can be determined using robust methods such as $[8,5]$. The second problem is that for the quaternion to represent a rotation its norm needs to be 1 . Replacing the norm constraints with the weaker constraint $\sum_{i}\left\|\mathbf{q}_{i}\right\|^{2}=n$ results in a lower bound of the original problem (since the minimization is carried out over a larger feasible set).

A simple heuristic to create a solution with the correct norms is to compute the eigen-solution and then modify the norms to be one. We will refer to this method as the normalized eigen-solution.

In this paper we propose an approach based on Lagrangian Duality to handle all the norm constraints. In general, problems with a large number of quadratic constraints are hard to solve and we cannot expect to always achieve an optimal solution. However in special cases Lagrangian duality has been shown to provide good solutions. For example in the case of one quadratic constraint it can be shown that the approach really finds the optimal solution [16].

\section{A Duality Based Approach}

In this section we present the theoretical foundation of our method. The key property making it possible to utilize this framework is that the function and 
the constraints are all quadratic functions. Therefore the dual problem can be computed in closed form. Furthermore, because we have only equality constraints we will be able to generate dual solutions efficiently.

\subsection{The Primal and Dual Problems}

Our goal is to solve what we refer to as the primal problem

$$
\begin{aligned}
& \min \sum_{i, j}\left\|Q_{i j} \mathbf{q}_{i}-\mathbf{q}_{j}\right\|^{2} \\
& \text { s.t } \mathbf{q}_{i}^{T} \mathbf{q}_{i}=1, i=0,1, \ldots, n
\end{aligned}
$$

To fix the coordinate system we will let one of the quaternions $\mathbf{q}_{0}$ be $\left[\begin{array}{llll}1 & 0 & 0 & 0\end{array}\right]^{T}$. This corresponds to letting the first absolute orientation be the identity matrix. We will stack all the remaining quaternions in a vector q. In matrix form we may now write the problem as

$$
\begin{gathered}
\min \|A \mathbf{q}-b\|^{2} \\
\text { s.t } \mathbf{q}^{T} N_{i} \mathbf{q}=1, i=1, \ldots, n .
\end{gathered}
$$

Here $A$ and $b$ are constructed from the relative rotation measurements, and $N_{i}$ is a matrix constructed such that $\mathbf{q}^{T} N_{i} \mathbf{q}=\mathbf{q}_{i}^{T} \mathbf{q}_{i}$.

The Lagrangian of this problem is

$$
\mathcal{L}(q, \lambda)=\|A \mathbf{q}-b\|^{2}+\sum_{i=1}^{n} \lambda_{i}\left(1-\mathbf{q}^{T} N_{i} \mathbf{q}\right) .
$$

The Lagrange multipliers $\lambda_{i}$ can be seen as a way of adding a penalty for unfulfilled constraints. Since $\mathcal{L}(\mathbf{q}, \lambda)=f(\mathbf{q})$ for all $\mathbf{q}$ feasible in (8) it is easy to see that the dual function

$$
d(\lambda)=\min _{\mathbf{q}} \mathcal{L}(\mathbf{q}, \lambda),
$$

is a lower bound of the optimal value of the primal problem. We now seek to find the largest possible lower bound

$$
\max _{\lambda} \min _{\mathbf{q}} \mathcal{L}(\mathbf{q}, \lambda) .
$$

It can be seen that the relaxation proposed by [6] corresponds to letting all the $\lambda_{i}$ be equal in the above optimization problem. Hence the result of (11) will always be at least as good as the eigen-solution. Since the Lagrangian is quadratic in $\mathbf{q}$ we can compute the dual problem in closed form. In block matrix form we write

$$
\max _{\lambda} \min _{\mathbf{q}}\left[\begin{array}{ll}
\mathbf{q}^{T} & 1
\end{array}\right] \mathcal{M}(\lambda, 0)\left[\begin{array}{l}
\mathbf{q} \\
1
\end{array}\right],
$$

where the matrix $\mathcal{M}(\lambda, 0)$ is given by

$$
\mathcal{M}(\lambda, \gamma)=\left[\begin{array}{cc}
A^{T} A-\sum_{i=1}^{n} \lambda_{i} N_{i} & -A^{T} b \\
-b^{T} A & b^{T} b+\sum_{i=1}^{n} \lambda_{i}-\gamma
\end{array}\right] .
$$


Further more we can homogenize the objective function in (12) by adding a scalar variable $q_{n+1}$ to the quadratic form and adding the extra dual variable $\gamma$. This gives

$$
\max _{\lambda, \gamma} \min _{\mathbf{q}, q_{n+1}}\left[\mathbf{q}^{T} q_{n+1}\right] \mathcal{M}(\lambda, \gamma)\left[\begin{array}{c}
\mathbf{q} \\
q_{n+1}
\end{array}\right]+\gamma
$$

If we minimize this expression with respect to $\mathbf{q}, q_{n+1}$ we see that the result will be unbounded unless $\mathcal{M}(\lambda, \gamma) \succeq 0$. Since we are interested in maximizing the lower bound we only need to consider $\mathcal{M}(\lambda, \gamma) \succeq 0$. It is easy to see that if $\mathcal{M}(\lambda, \gamma) \succeq 0$ then the minimum of (14) is going to be $\gamma$. Hence we should find the maximum $\gamma$ such that $\mathcal{M}(\lambda, \gamma) \succeq 0$,. Therefore our dual problem is going to be

$$
\begin{array}{cl}
\max _{\gamma, \lambda} & \gamma \\
\text { s.t } & \mathcal{M}(\lambda, \gamma) \succeq 0 .
\end{array}
$$

This is a linear semi definite program which can be solved using standard solvers. It provides a lower bound on the optimal value of the primal problem. This lower bound can be used to estimate the quality of the primal solution, and in certain cases even verify optimality of a solution. Specifically, suppose that we have a feasible dual solution and a feasible primal solution. If these yield the same value when inserting into the primal and dual programs respectively, then they are both optimal.

For general problems we cannot hope that the lower bound given by the dual program is going to attain the same value as the primal solution unless we have some convexity properties. The difference between these values are called the duality gap. There are however a few exceptions, most notably, for a quadratic function with a single quadratic constraint, the duality gap is zero [16]. We will show experimentally that in our case the gap is always zero unless the system is subjected to severe noise.

\subsection{Generating Primal and Dual Solutions}

Next we deal with the problem of generating good primal and dual solutions. We propose two ways of doing this; Either by solving the semi definite program, or by locally optimizing the primal problem and computing dual variables from the resulting solution. Solving the semi definite program is the safest way of finding the best dual variables, however for large scale problems it is costly and therefore we also propose a method based on local optimization that greatly improves the efficiency of the scheme.

To generate primal solutions using the semidefinite program we first make use of the "dual of the dual". Using methods similar to what we did in the 
previous section it is possible to derive the program

$$
\begin{gathered}
\min \operatorname{tr}\left(\left[\begin{array}{cc}
A^{T} A & -A^{T} b \\
-b^{T} A & b^{T} b
\end{array}\right]\left[\begin{array}{cc}
Z & z \\
z^{T} & 1
\end{array}\right]\right) \\
\text { s.t. } \operatorname{tr}\left(N_{i} Z\right)=1, i=1, \ldots, n \\
{\left[\begin{array}{ll}
Z & z \\
z^{T} & 1
\end{array}\right] \succeq 0 .}
\end{gathered}
$$

which is dual to (15)-(16). It is easy to see that if this problem provides a solution matrix with rank one then this matrix can be factored as $\mathbf{q} \mathbf{q}^{T}$ which provides a solution for the original primal problem. Since there is no duality gap between the semi definite programs [17] there will be no gap between any of the problems, should this happen. We will show experimentally that unless the noise level is very large the result of the above optimization is a rank one solution.

For large scale problems it can be to expensive to solve the semi definite program. Therefore we propose to use local optimization to generate a candidate primal solution from which a dual solution can be computed. The key observation is that in case there is no duality gap then the lower bound is tight. Hence there are $\lambda$ such that

$$
\min _{q}\|A \mathbf{q}-b\|^{2}+\sum_{i=1}^{n} \lambda_{i}\left(1-\mathbf{q}^{T} N_{i} \mathbf{q}\right)
$$

gives the same result as the primal problem. Taking the gradient with respect to $\mathbf{q}$ gives

$$
2 A^{T} A \mathbf{q}-2 A^{T} b-\sum_{i=1}^{n} \lambda_{i} 2 N_{i} \mathbf{q}=0 .
$$

Since we have only equality constraints we have no further constraints on the dual variables and therefore this is a linear system of equations in $\lambda_{i}$. That is, given a candidate solution $\mathbf{q}$ we can compute a set of dual variables $\lambda$ by solving a linear set of equations. Assuming that there are more equations than the number of quaternions/constraints this is going to be an over determined system. However for a local minimum of the primal problem they are guaranteed to have an exact solution, since these are also necessary conditions for local minima. The final dual variable $\gamma$ is obtained from the objective value of the primal problem.

\subsection{Algorithm}

In summary, for large scale problems we propose the following algorithm

1. Compute and normalize the eigen-solution (2).

2. Starting from the normalized eigen-solution locally optimize (5)-(6).

3. From the local optimum compute the dual solution (21).

4. Insert the dual variables into (16). If $\mathcal{M}(\lambda, \gamma)$ is positive semi definite then the primal solution is globally optimal. 
To determine the correct signs of the quaternions we first use the robust method of $[8]$.

In case there is a duality gap $\mathcal{M}(\lambda, \gamma)$ will not be positive semi definite. In this case it is still possible to obtain a lower bound on the optimal value by lowering $\gamma$ until $\mathcal{M}(\lambda, \gamma)$ is positive semi definite. However this situation has not occurred during any of our real experiments.

For local optimization we use a straight forward Levenberg-Marquardt algorithm. To linearize the residuals we compute a basis for the tangent plane at each quaternion in the current solution, and consider the function on these tangent spaces instead. This gives the first order Taylor expansion of the residuals.

\section{Experiments}

In this section we evaluate our proposed methods. We first use synthetic data to test the quality of the proposed relaxation for various levels of noise. We then evaluate the speed of the method for varying problem size. Finally we test the method on a number of data sets from real applications.

\subsection{Synthetic Data}

First we generate 100 random rotation matrices and convert these to quaternion forms. To these rotations we add Gaussian noise. We then set up the primal system (5). Figure 1 shows the error in $L_{2}$ norm of the eigen-solution, the normalized eigen-solution, the local optimization method of the primal problem and the dual solution for noise levels in the interval $[0,1 \pi]$. When all relative rotations are available there is almost no difference between the methods. Since images that are far away from each other often have few corresponding points, we also try removing pairwise connections with an angle difference greater than $\pi / 8$. If the rotation graph gets partitioned into several connected subsets when doing this we only use the part that contains the first rotation. Figure 1 shows the results for this setup. It can be seen that the eigen solution gives a weaker lower bound than the dual solution. Furthermore, the normalized eigen-solution does not give a globally optimal solution when the noise increases. Note however that differences are only visible at extreme amounts of noise. For reasonable amounts (less than $0.1 \pi$ ) all four solutions give roughly the same result.

To explore the time complexity we change the size of the system while keeping a constant noise level. The run times for the different algorithms are displayed in Figure 2. The difference between the local optimization method and the normalized eigen-solution is very small while the time for solving the dual problem grows much faster with an increase of the number of rotations.

\subsection{Real Experiments}

Next we consider a few real experiments. As we saw in the previous section the local optimization procedure starting from the normalized eigen-solution provides verifiable globally optimal solutions unless the noise levels are severe. Here 

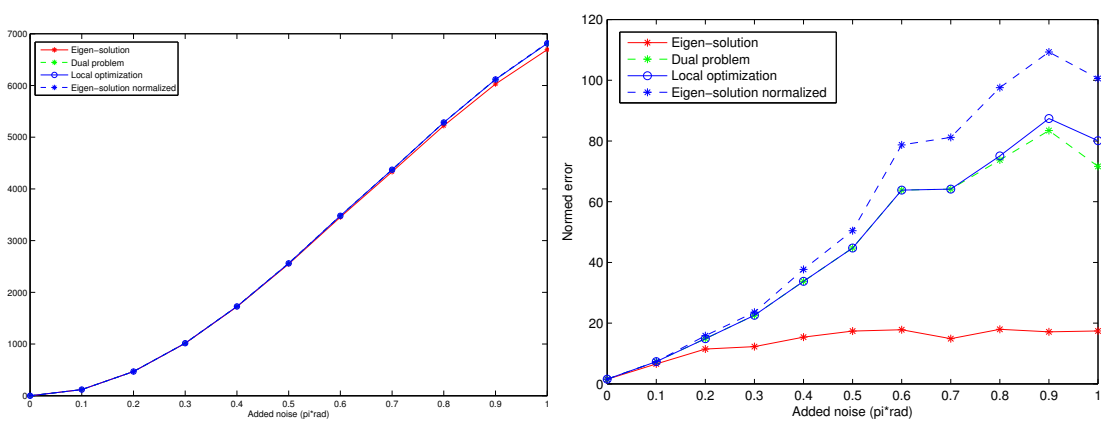

Fig. 1. Left: The error for 100 random matrices in the $L_{2}$ norm, for the different methods. Right: The error in $L_{2}$ for 100 random matrices when we only allow connections between matrices with angle difference less than $\pi / 8$.

we will first present some real experiments where the noise levels are low and see that the same holds true. Figure 3 shows two panoramas that were created using the proposed method. First pairwise relative rotations are computed robustly (using RANSAC) for every pair of images that have corresponding points. Then we use rotation averaging to compute the absolute orientations of the cameras. Table 1 shows the results. Here the noise level is very low and the normalized eigen-solution can be seen to give exactly the same value as the global solution.

Table 1. Resulting objective function values for the outlier free datasets.

\begin{tabular}{ccccc}
\hline Data set & Eigen-Sol. Normalized Eigen-Sol. Locally Optimal Sol. Lower Bnd \\
\hline Niagara & 0.001722 & 0.001723 & 0.001723 & 0.001723 \\
Toronto & 0.000220 & 0.000220 & 0.000220 & 0.000220 \\
Cathedral & 54.86 & 54.87 & 54.87 & 54.87
\end{tabular}

Figure 4 shows a 3D reconstruction where the camera orientations were computed with the proposed method. The relative rotations were computed from pairwise epipolar geometry estimates. To remove potential outliers and determine the signs we applied the approach outlined in [8]. After computing the absolute orientations we used these to estimate structure and camera positions and applied bundle adjustment to the result. To the right in Figure 4 are the computed normals for the eigen-solution. It can be seen that they are very close to being one, and the values in Table 1 show that the normalized eigen-solution is very close to optimal (it is correct up to the fourth decimal). The local improvement converges in one iteration and yields the same value as the lower bound. To compute the result takes 12.89 seconds. Out of this 0.85 are spent computing the eigen-solution, 2.61 are spent on computing the local optimizer 


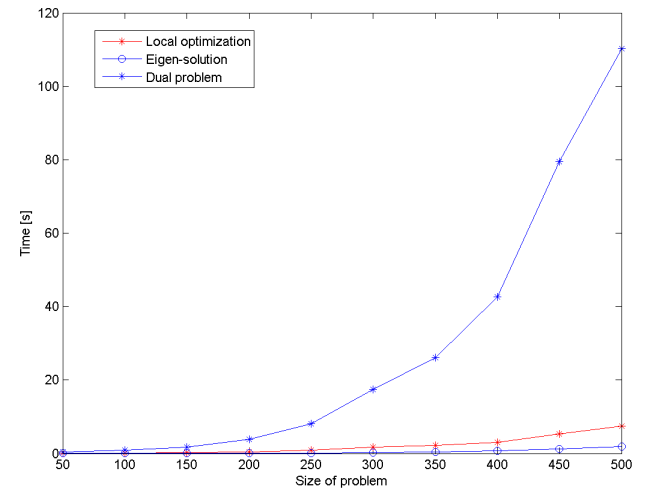

Fig. 2. The run time in seconds for the eigen-solution, the local optimization and the dual problem, for different numbers of rotations.



Fig. 3. Panoramas of Niagara Falls (created from 15 images with 63 relative rotations) and Toronto (created from 24 images with 127 relative rotaions).

and verifying that this one is globally optimal and the rest is spent on setting up the problem. The obtained absolute orientations are on average 0.86 degrees from the final ones obtained from bundle adjustment, the maximal difference is 2.3 degrees.

In the previously studied experiments the noise levels are small enough for the normalized eigen-solution to achieve near global optimality. There are however cases where the noise levels are substantially larger, namely in the presence of outlier rotations. In this case it cannot be argued that the $L_{2}$ norm is a good choice since this type of noise is typically not Gaussian. Still we argue that being able to solve the $L_{2}$ norm optimally is of value since methods such as iterative reweighted least squares (IRLS), can be utilized to solve a large number of robust functions $[18,19]$.

Next we will apply our framework in a re-weighted least squares approach for rotation averaging. Since our relative rotations contain both noise and outliers 

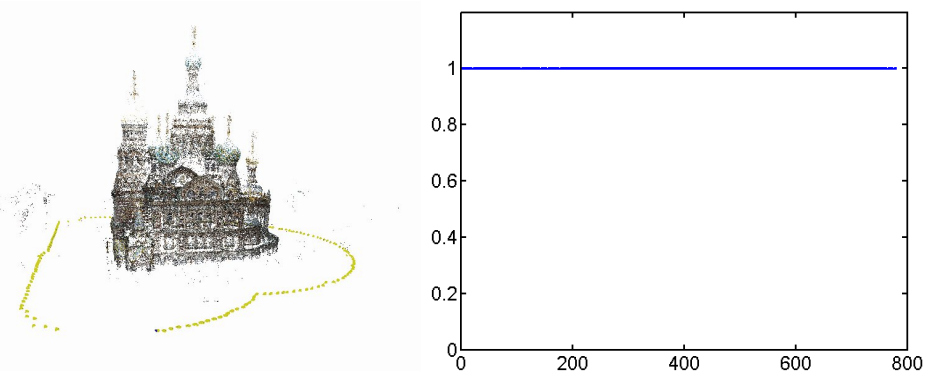

Fig. 4. Left: Reconstruction of a Cathedral in St. Petersburg, 781 cameras and 74004 relative rotation estimates. Right: The norms of the eigen-solution.

we use the robust cost function

$$
\sum_{i j} \frac{\left\|Q_{i j} \mathbf{q}_{i}-\mathbf{q}_{j}\right\|^{2}}{\sqrt{\left\|Q_{i j} \mathbf{q}_{i}-\mathbf{q}_{j}\right\|^{2}+\epsilon^{2}}}
$$

Figure 5 shows the graph of a one dimensional version. Here $\epsilon$ was chosen to be 0.01 since this is what we use in all our experiments. If the residual $\left\|Q_{i j} \mathbf{q}_{i}-\mathbf{q}_{j}\right\|$ is small compared to $\epsilon$ then the function is roughly quadratic and when $\| Q_{i j} \mathbf{q}_{i}-$ $\mathbf{q}_{j} \|$ grows it approaches the $L_{1}$-norm which is known to be robust to outliers.

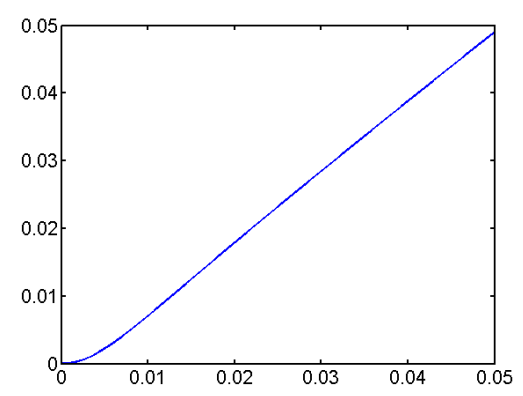

Fig. 5. The function $f(t)=\frac{t^{2}}{\sqrt{t^{2}+\epsilon^{2}}}$ for $\epsilon=0.01$.

To optimize (22) we employ an iterative re-weighted least squares approach. For a given solution $\mathbf{q}^{k}$ we compute the weights

$$
w_{i}^{k}=\frac{1}{\sqrt{\left\|Q_{i j} \mathbf{q}_{i}^{k}-\mathbf{q}_{j}^{k}\right\|^{2}+\epsilon^{2}}} .
$$


To obtain the next iterate $\mathbf{q}^{k+1}$ we optimize the modified primal problem

$$
\begin{aligned}
& \min \left\|\sqrt{W^{k}}(A \mathbf{q}-b)\right\|^{2} \\
& \text { s.t } \mathbf{q}^{T} N_{i} \mathbf{q}=1, i=0,1, \ldots, n,
\end{aligned}
$$

where $\sqrt{W^{k}}$ is a diagonal matrix containing the square root of the coefficients $w_{i}^{k}$. (Note that they are repeated four times since each equation $Q_{i j} \mathbf{q}_{i}-\mathbf{q}_{j}=0$ gives rise to four rows in $A$ and $b$.) It is easily seen that the theory of Section 3 applies to this problem as well. In the first iteration we let $\sqrt{W^{0}}$ be the identity matrix, and start our local optimization from the normalized linear solution. In the next iteration we use the previously optimal solution as a starting point.

Figures 6-9 and Table 2 shows the results when applying our approach to 4 data sets. To the left are the reconstructions obtained from the estimated absolute orientations (as described above). To the right in the figures are the norms of the eigen-solution used as a starting solution for the IRLS. It can be seen that the noise levels are higher here and in some cases the eigen-solution gives a much weaker lower bound. In particular for the larger data sets the norms of some of the quaternions can be far from one. Surprisingly, after normalizing, the obtained solution is very close to optimal as can be seen from our stronger lower bound. In all cases we were able to verify global optimality (24)-(25) after applying a local optimization from these starting points.

Table 2. Results for the 4 data sets after the first iteration of the IRLS algorithm.

\begin{tabular}{ccccc}
\hline Dataset: & Eigen-Sol. Normalized Eigen-Sol. Local Opt. Lower bound \\
\hline Hall Building & 34.6 & 35.17 & 35.12 & 35.12 \\
Monastery & 2021 & 2227 & 2226 & 2226 \\
Kazan & 8366 & 8813 & 8812 & 8812 \\
Alcatraz & 20489 & 22242 & 22236 & 22236 \\
\hline
\end{tabular}

Table 3. Running times, the number of times (24)-(25) is solved and the total number of Levenberg-Marquardt updates made in the IRLS algorithm, when running on the 4 data sets.

\begin{tabular}{cccc}
\hline Dataset: & Running time (s) Nr. times (24)-(25) & solved Nr. of LM updates. \\
\hline Hall Building & 1.6 & 11 & 30 \\
Monastery & 70 & 55 & 163 \\
Kazan & 73 & 18 & 146 \\
Alcatraz & 743 & 28 & 840 \\
\hline
\end{tabular}

Table 3 shows the execution times and the number of iterations required for the IRLS method to converge. While the number of times the modified primal 

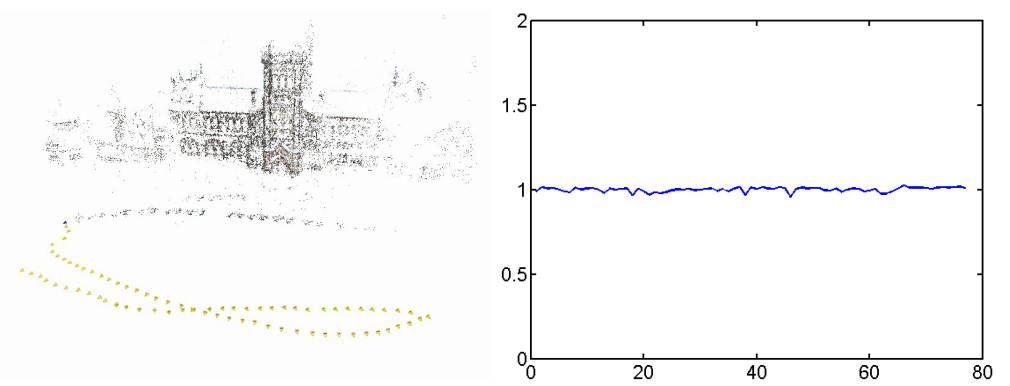

Fig. 6. Left: Reconstruction of the Hall building, 77 cameras and 1478 relative rotation estimates. Right: The norms of the eigen-solution.

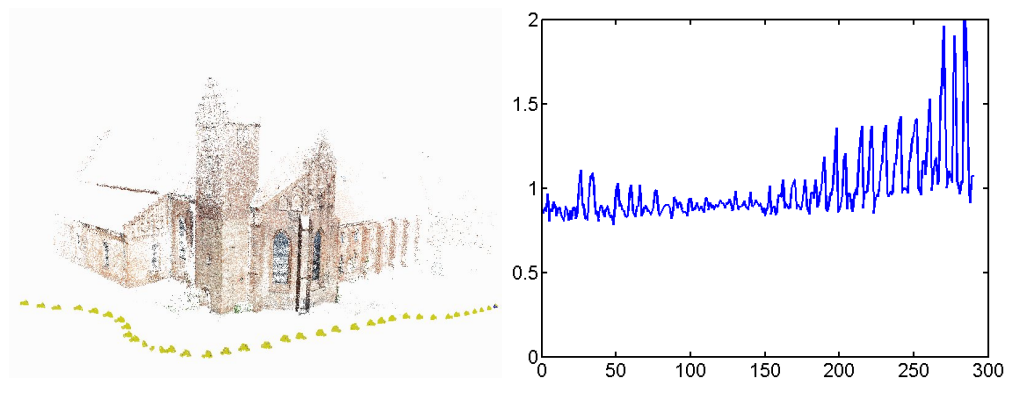

Fig. 7. Reconstruction of Ystad Monastery, 290 cameras and 21463 relative rotation estimates. Right: The norms of the eigen-solution.

problem is solved (24)-(25) does not appear to depend on the size of the problem, the number of local updates (Leveberg-Marquardt steps) increases with the size of the problem. Typically most of these iterations are spent in the first step of the algorithm. Each time we solved (24)-(25) the solution was verified to be optimal.

\section{Conclusions}

We have presented a semi definite programming framework for solving the multiple rotation averaging problem. Our method attempts to solve the linear system from [6] while still enforcing the norm-constraints of the quaternions. We showed that unless the noise level is severe local optimization starting from the normalized eigen-solution is sufficient for obtaining the global optimum. Based on this we showed how to verify that a local optimal solution is also global resulting in significant speedups compared to solving the semi definite program. 

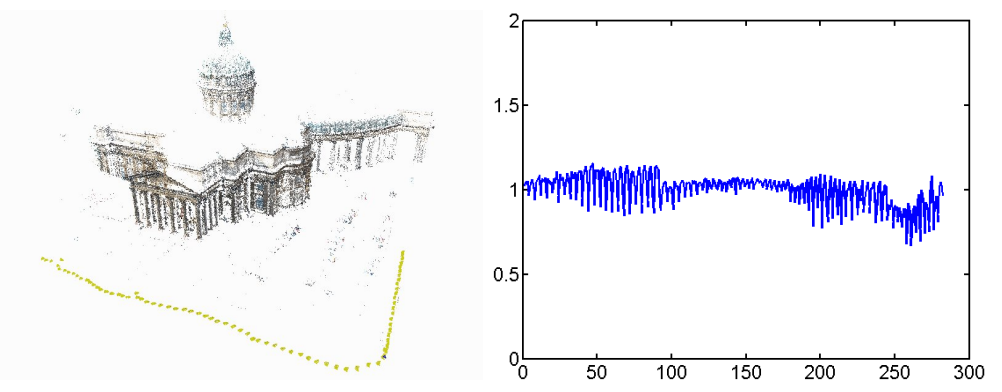

Fig. 8. Left: Reconstruction of the Kazan Cathedral, St. Petersburg, 282 cameras and 35685 relative rotation estimates. Right: The norms of the eigen-solution.

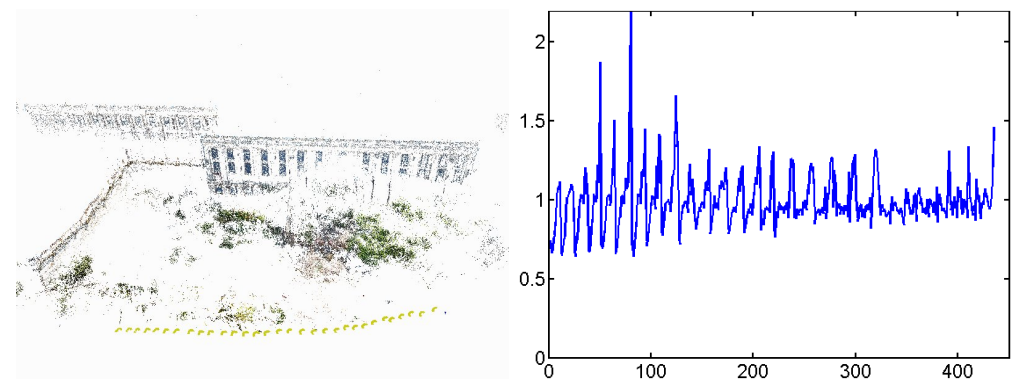

Fig. 9. Reconstruction of the west side of Alcatraz, 435 cameras and 70148 relative rotation estimates. Right: The norms of the eigen-solution.

\section{Acknowledgements}

This work was funded by the Swedish Foundation for Strategic Research through the programmes Future Research Leaders and Wear- able Visual Information Systems, the European Research Council through the programme GlobalVision and the Swedish strategic research project ELLIIT.

\section{References}

1. Nistér, D.: An efficient solution to the five-point relative pose problem. Trans. Pattern Analysis and Machine Intelligence (2004)

2. Tardif, J.P., Bartoli, A., Trudeau, M., Guilbert, N., Roy, S.: Algorithms for batch matrix factorization with application to structure-from-motion. In: Conf. Computer Vision and Pattern Recognition. (2007)

3. Gherardi, R., Farenzena, M., Fusiello, A.: Improving the efficiency of hierarchical structure-and-motion. In: Conf. Computer Vision and Pattern Recognition. (2010)

4. Crandall, D., Owens, A., Snavely, N., Huttenlocher, D.P.: Discrete-continuous optimization for large-scale structure from motion. In: Proc. IEEE Conf. on Computer Vision and Pattern Recognition. (2011) 
5. Enqvist, O., Kahl, F., Olsson, C.: Non-sequential structure from motion. In: Workshop on Omnidirectional Vision, Camera Networks and Non-Classical Cameras, Barcelona, Spain (2011)

6. Govindu, V.: Combining two-view constraints for motion estimation. In: Conf. Computer Vision and Pattern Recognition. (2001)

7. Govindu, V.: Lie-algebraic averaging for globally consistent motion estimation. In: Conf. Computer Vision and Pattern Recognition. Volume I., Washington DC, USA (2004) 684-691

8. Govindu, V.: Robustness in motion averaging. In: Eur. Conf. Computer Vision. (2006)

9. Martinec, D., Pajdla, T.: Robust rotation and translation estimation in multiview reconstruction. In: Conf. Computer Vision and Pattern Recognition. (2007)

10. Hartley, R., Aftab, K., Trumpf, J.: L1 rotation averaging using the weiszfeld algorithm. In: Conf. Computer Vision and Pattern Recognition, Colorado Springs, USA (2011)

11. Hartley, R., Trumpf, J., Dai, Y.: Rotation averaging and weak convexity. In: International Symposium on Mathematical Theory of Networks and Systems, Piscataway, USA (2010)

12. Dai, Y., Trumpf, J., Li, H., Barnes, N., Hartley, R.: Rotation averaging with application to camera-rig calibration. In: Asian Conf. on Computer Vision. (2009)

13. Zach, C., Klopschitz, M., Pollefeys, M.: Disambiguating visual relations using loop constraints. In: Conf. Computer Vision and Pattern Recognition. (2010)

14. Sharp, G., Lee, S., Wehe, D.: Multiview registration of $3 \mathrm{~d}$ scenes by minimizing error between coordinate frames. Trans. Pattern Analysis and Machine Intelligence 26 (2004) 1037-1050

15. Hartley, R., Seo, Y.: Verifying global minima for $L_{2}$ minimization problems. In: Conf. Computer Vision and Pattern Recognition, Anchorage, USA (2008)

16. Sorensen, D.: Minimization of a large-scale quadratic fuction subject to a spherical constraint. SIAM J. Optim. 7 (1997) 141-161

17. Boyd, S., Vandenberghe, L.: Convex Optimization. Cambridge University Press (2004)

18. Daubechies, I., DeVore, R., Fornasier, M., Güntürk, C.S.: Iteratively reweighted least squares minimization for sparse recovery. Communications on Pure and Applied Mathematics 63 (2010) 1-38

19. Bube, K.P., Langan, R.T.: Hybrid 11 / 12 minimization with applications to tomography. Geophysics 62 (1997) 1183-1195 